\title{
Water Impact Analysis Based on the Trajectory-Tracking Method
}

\author{
WANG Yonghu, HU Wei ${ }^{a}$ and CHEN Caihua \\ Civil Aviation Flight University of China, Guanghan city Sichuan Province, China, 618307 \\ a huweiyes@yeah.net
}

Keywords: Water impact, Trajectory-tracking method, impact loads, Movement clearance.

Abstract. The motions were repeatedly filmed by a high-speed camera when the rigid structure vertically dropped above water surface and then digital image processing was carried out based on trajectory-tracking method. Three key points on the wedge were tracked automatically, generating the corresponding discrete measurements of the progressive displacements. The acceleration-time curves were got by the given algorithm. The comparisons of the velocity and acceleration time histories were implemented for the satisfactory data processing way. At last, the relative motion between the wedge and top structure were analyzed based on the method. The results can not only be a validation of the data from the traditional approach and numerical simulation, but also check the experiment further.

\section{Introduction}

Water impact, together with water ditching, is a nonlinear phenomenon in engineering. The study was proposed about eight decades ago, yet there has not been a mathematical model capable of accurate predictions. The difficulty derives from the complexity of the system which mainly includes the motion of a body, fluid-structure interaction and fluid surface fluctuation.

Three methods have been developed in the measurement of an impact of a rigid body onto water: 1) accelerometer is adopted to obtain the measurements of acceleration data set. The velocity and displacement profiles are obtained from the acceleration $[1,2,3]$; 2) the velocity of a rigid body is accurately measured by an optical interferometer [4]. The acceleration and displacement are calculated from the velocity [5]; 3) the displacement is obtained by the images of a high-speed camera, which was also adopted in this paper. The velocity and acceleration are calculated from the displacement.

\section{Tracking method and algorithm}

In the water impact experiment, the bottom of impact structures must be in-depth thought in order to obtain the maximum impact load. In the present study, the impact structure comprised of the bottom structure and the top structure. The top one was a fixture to link the bottom one which was wedge specimen or other specimen. The wedge specimen used in this experiment was shown in Fig. 1.

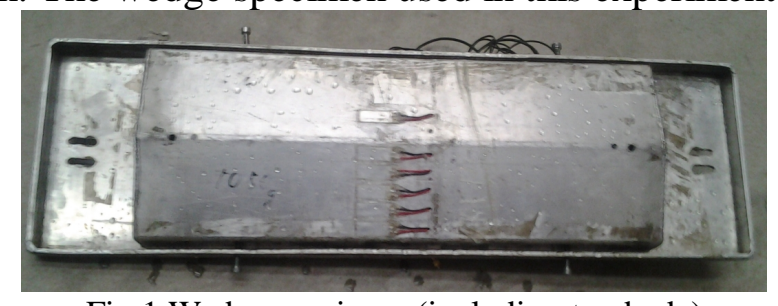

Fig.1 Wedge specimen (including top body)

The process of impact on the clam water was recorded by a Phantom V711 digital high-speed camera. The selected frame rate was 10,000 per second. The pixel coordinates of the center of specific points, which was represented by the centeroid of the black area, can be gained manually.

The result directly came from the calculation of discrete displacement data set. The velocity, $v_{n}$, at a given time $t_{n}$ is obtained by calculating the distance moves between $t_{n-1}$ and $t_{n+1}$, and dividing that distance by the immutable time gap, $\Delta t$.

$$
v_{n}=\frac{\Delta D}{\Delta T}=\frac{d_{n+1}-d_{n-1}}{t_{n+1}-t_{n-1}}=\frac{d_{n+1}-d_{n-1}}{2 \Delta t}
$$


The acceleration $a_{n}$ is determined through the five discrete displacements from $d_{n-2}$ to $d_{n+2}$ consistently. The equation is

$$
a_{n}=\frac{d_{n-2}-d_{n-1}-2 d_{n}-d_{n+1}+2 d_{n+2}}{7\left(t_{n}-t_{n-1}\right)^{2}}=\frac{d_{n-2}-d_{n-1}-2 d_{n}-d_{n+1}+2 d_{n+2}}{7 \Delta t^{2}}
$$

The Mean Squared Error (MSE) between the polynomial approximation and the original data set is the obtained according to the equation as below

$$
M S E=\frac{1}{n} \sum_{i=1}^{n}\left(x_{p}-x_{o}\right)^{2}
$$

where, $x_{p}$ is the polynomial approximation data, $x_{0}$ is the original data.

\section{Results and discussions}

According to the experimental image sequences, three specific points were marked in every image to generate displacement data sets of the motion. And the acceleration results were obtained based on the above algorithm. The average displacement data set was gained from the three data sets. Four acceleration profiles were achieved from the three displacement data sets. Fig.2 depicts the acceleration curves in which the high-frequency samples were filtered. Here the appropriate time step was selected in order to avoid the improper sample frequency, hereafter the good method of filtering technology was given to deal with the sample data.

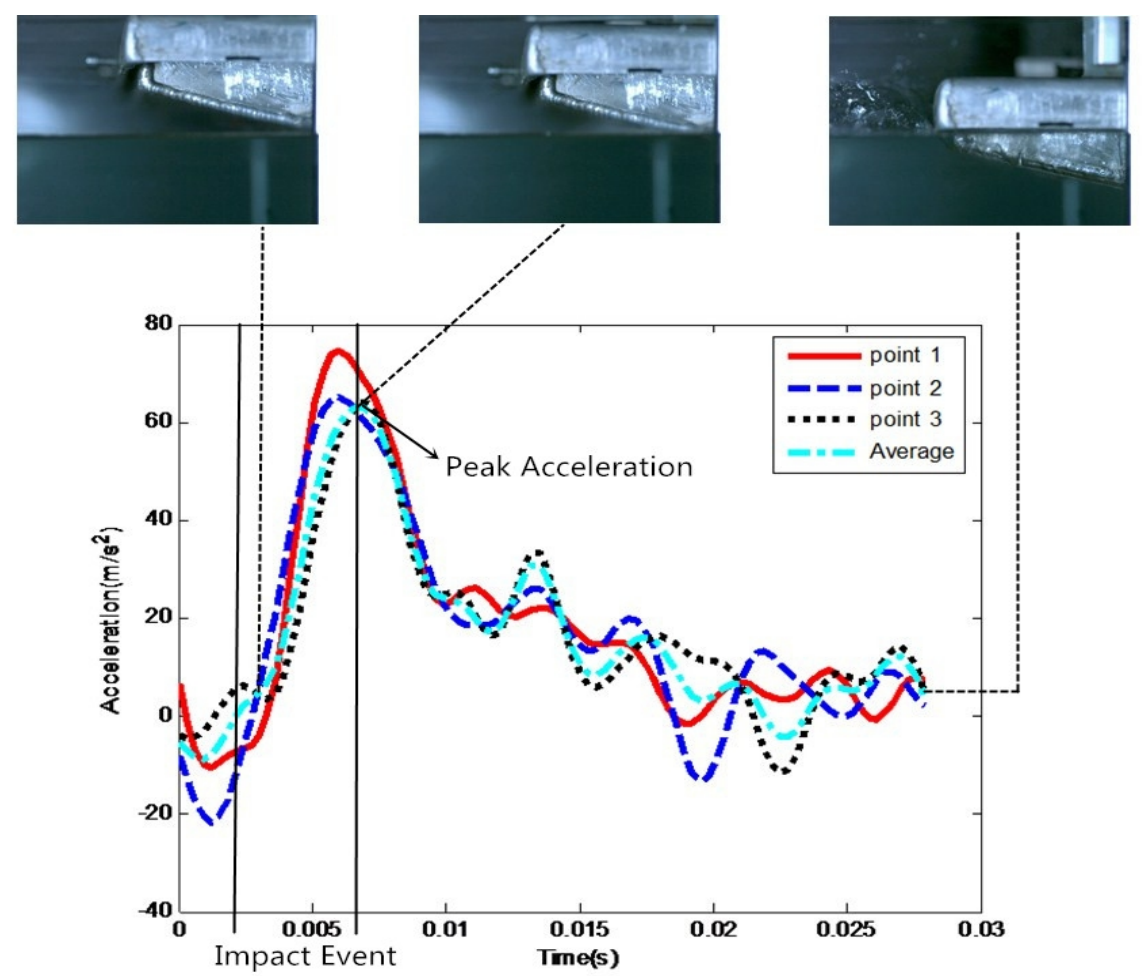

Fig.2 Acceleration-time history

The maximum acceleration and duration of impact were focused in the water impact domain. The time period marked the impact event in the figure is important basement for the analysis of impact load and duration. The initial and end point of the impact event were respectively zero and the peak value of acceleration. Besides the double duration of impact event, three acceleration-time curves fluctuated dramatically, but the acceleration curve which was obtained by the average displacement data set was smoother. It indicated that the adopting average displacement data set was useful in reducing error in order to approach to the realistic impact event. The maximum accelerations were $363 \mathrm{~m} / \mathrm{s}^{2}, 342 \mathrm{~m} / \mathrm{s}^{2}$, and $340 \mathrm{~m} / \mathrm{s}^{2}$ respectively and the average value is $348 \mathrm{~m} / \mathrm{s}^{2}$. So here the data set between $3 \mathrm{~ms}$ and $8.7 \mathrm{~ms}$ was selected for following analysis.

The comparison between the first derivative of polynomial approximation of velocities based on the average displacement data set over the impact event and the polynomial approximation of accelerations 
was carried out. The resulting polynomial approximations of the velocity-time and acceleration-time history are shown in Fig.4 and Fig.5.

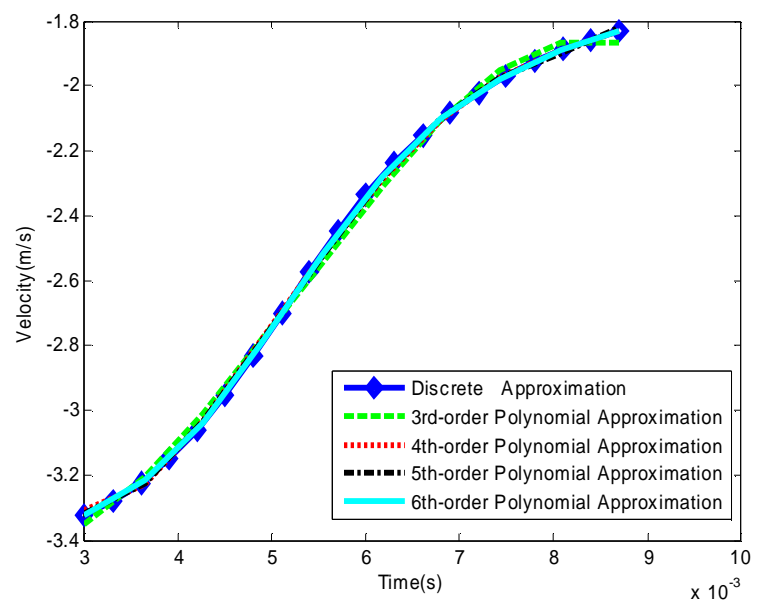

Fig.3 Polynomial approximations of velocity values

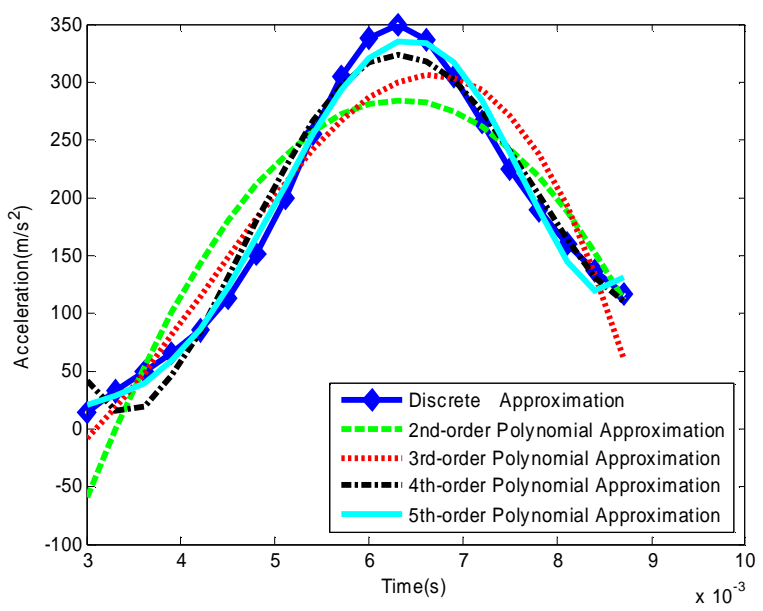

Fig.4 Polynomial approximations of acceleration values

Table .1 depicts the velocity-time histories, the first derivations of them and Mean Squared Error. Table. 2 depicts the acceleration-time histories and the Mean Squared Error. From the Mean Squared Error in the two tables, the minimum Mean Squared Error were obtained in the 6th-order polynomial approximation of the velocity function and in the 5th-order of the acceleration function over the impact zone respectively. It means that the method is adoptable in the drop run.

Table 1: Characteristics of polynomial approximations of velocity of the drop run

\begin{tabular}{|c|c|c|c|}
\hline & Function & $\begin{array}{c}\text { Mean } \\
\text { Squared } \\
\text { Error }\end{array}$ & First Derivation of Function \\
\hline $\begin{array}{c}\text { 3rd-order Polynomial } \\
\text { Approximation }\end{array}$ & $\left(-1.3142 \mathrm{t}_{\mathrm{n}}{ }^{3}+0.0208 \mathrm{t}_{\mathrm{n}}{ }^{2}-0.0001 \mathrm{t}_{\mathrm{n}}\right) * 10^{7}$ & 0.0231 & $\left(-3.9426 t_{n}{ }^{2}+0.0416 t_{n}-0.0001\right) * 10^{7}$ \\
\hline $\begin{array}{c}4 \text { th-order Polynomial } \\
\text { Approximation }\end{array}$ & $\left(3.7050 \mathrm{t}_{\mathrm{n}}{ }^{4}-0.0998 \mathrm{t}_{\mathrm{n}}{ }^{3}+0.0009 \mathrm{t}_{\mathrm{n}}{ }^{2}\right) * 10^{9}$ & 0.0071 & $\left(14.82 t_{n}{ }^{3}-0.2994 t_{n}{ }^{2}+0.0018 t_{n}\right) * 10^{9}$ \\
\hline $\begin{array}{c}\text { 5th-order Polynomial } \\
\text { Approximation }\end{array}$ & $\left(5.1358 \mathrm{t}_{\mathrm{n}}{ }^{5}-0.1132 \mathrm{t}_{\mathrm{n}}{ }^{4}+0.0007 \mathrm{t}_{\mathrm{n}}{ }^{3}\right) * 10^{11}$ & 0.0055 & $\left(25.679 t_{n}{ }^{4}-0.4528 t_{n}{ }^{3}+0.0021 t_{n}{ }^{2}\right)^{*} 10^{11}$ \\
\hline $\begin{array}{c}\text { 6th-order Polynomial } \\
\text { Approximation }\end{array}$ & $\left(-4.3258 \mathrm{t}_{\mathrm{n}}{ }^{6}+0.1570 \mathrm{t}_{\mathrm{n}}{ }^{5}-0.0023 \mathrm{t}_{\mathrm{n}}{ }^{4}\right) * 10^{14}$ & 0.0011 & $\left(25.9548 t_{n}{ }^{5}+0.785 t_{n}{ }^{4}-0.0092 t_{n}{ }^{3}\right) * 10^{14}$ \\
\hline
\end{tabular}

Table 2: Characteristics of polynomial approximations of acceleration of the drop run

\begin{tabular}{|c|c|c|}
\hline & Function & Mean Squared Error \\
\hline $\begin{array}{c}\text { 2nd-order Polynomial } \\
\text { Approximation }\end{array}$ & $\left(-3.0851 \mathrm{t}_{\mathrm{n}}{ }^{2}+0.0391 \mathrm{t}_{\mathrm{n}}-0.0001\right) * 10^{7}$ & 41.9919 \\
\hline $\begin{array}{c}\text { 3rd-order Polynomial } \\
\text { Approximation }\end{array}$ & $\left(-6.5543 \mathrm{t}_{\mathrm{n}}{ }^{3}+0.0842 \mathrm{t}_{\mathrm{n}}{ }^{2}-0.0002 \mathrm{t}_{\mathrm{n}}\right) * 10^{9}$ & 32.7467 \\
\hline $\begin{array}{c}4 \text { th-order Polynomial } \\
\text { Approximation }\end{array}$ & $\left(4.6105 \mathrm{t}_{\mathrm{n}}{ }^{4}-0.1144 \mathrm{t}_{\mathrm{n}}{ }^{3}+0.0010 \mathrm{t}_{\mathrm{n}}{ }^{2}\right) * 10^{12}$ & 17.9468 \\
\hline $\begin{array}{c}\text { 5th-order Polynomial } \\
\text { Approximation }\end{array}$ & $\left(1.5845 \mathrm{t}_{\mathrm{n}}{ }^{5}-0.0417 \mathrm{t}_{\mathrm{n}}{ }^{4}+0.0004 \mathrm{t}_{\mathrm{n}}{ }^{3}\right) * 10^{15}$ & 11.5454 \\
\hline
\end{tabular}

In order to reduce experimental error and ensure the accuracy of results, here the relative motion between wedge and wedge top structure was given by the trajectory tracking method. From the two displacement data sets shown as Fig. 5, they do not match well with each other. The fist reason for the disparity was they were not connected tightly, and the latter one was due to the friction between the structures and air compression which absorbed energy. It is helpful to improve the experimental facility and meet the experimental requirement. 


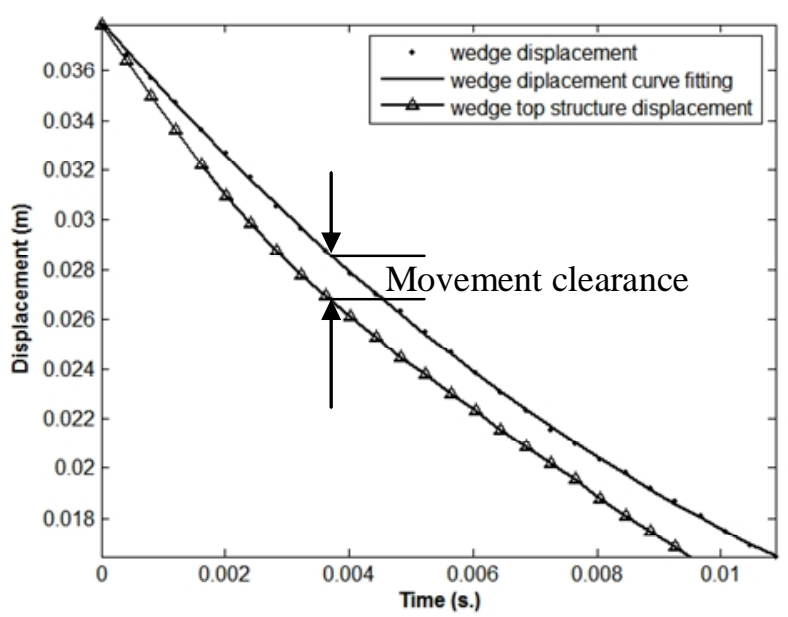

Fig.5 Relative motion between wedge and top structure

\section{Conclusions}

Water impact problem was analyzed based on the trajectory-tracking method. The progression of a wedge impacting into water was tested and filmed by the high-speed camera. The displacement data sets were obtained automatically from the imagines and velocities and acceleration time histories based on the given algorithm. Particular attention was given to the acceleration-time history based on the average displacement data set. It can be inferred from the Mean Squared Error that the 6th-order polynomial approximations of the velocity function and the 5th-order polynomial approximations of the acceleration function over the impact zone were optimal. The trajectory-tracking method is applied to deeply study water impact experiment and find the error because of the relative motion between the two bodies. The results and approach can act as references to the qualitative validation and comparison for the further numerical simulations.

\section{Acknowledgements}

The funding provided by the Joint Funds of the National Natural Science Foundation of China (Grant No.U1333133), the General Program of CAFUC (Grant No.J2011-03, Grant No. J2014-04) and the Open Fund Program of Flight Technology and Flight safety research base (Grant No.F2013KF02) in support for the project is greatly acknowledged.

\section{References}

[1] Brooks, J. R., Anderson, L. A., Dynamics of a Space Module Impacting Water: Journal of Spacecraft Rockets, Vol.31 (1994), pp.509-515

[2] Seddon, C. M., Moatamedi, M., Review of Water Entry with Applications to Aerospace Structures, International Journal of Impact, Vol.32 (2006), pp.1045-1067

[3] Rosellini L, Hersen F, Clanet C, Bocquet L, Skipping Stones: Journal of Fluid Mechanics, Vol.543, (2005), pp.137-146.

[4] R. Araki, A. Takita, et al, Impact Force Measurement of a Spherical Body Dropping onto a Water Surface, Review of Scientific Instruments, Vol.85 (2014), pp. 075108 1-5.

[5] Gekle S, Gordillo JM, van der Meer D, Lohse D. Supersonic Air Flow due to Solid-liquid Impact: Physical Review Letters, Vol.104 (2010), pp.245 